\title{
Medication Adherence and Its Determinants among Women of Cardiovascular Disease of Tertiary Care Hospital, Hyderabad
}

\author{
Haritha Arnipalli ${ }^{1}$ Sudha Bala ${ }^{1}$ Harshal Pandve ${ }^{1}$ \\ ${ }^{1}$ Department of Community Medicine, Employee's State Insurance \\ Corporation Medical College, Hyderabad, Telangana, India
}

\author{
Address for correspondence Sudha Bala, MBBS, MD (Community \\ Medicine), Flat No. 301, 2-2-1075/A, Shubodaya enclave, \\ Baghamberpet, Hyderabad-500013, Telangana, India \\ (e-mail: dr.sudhabala78@gmail.com).
}
Abstract
Keywords
- medication adherence
- the Morisky scale
- women

Background and Aim Adherence to long-term therapy for chronic illnesses in developed countries averages to $50 \%$. In developing countries, the rates are even lower. Poor adherence to long-term therapies severely compromises the effectiveness of treatment making this a critical issue in population health both from the perspective of quality of life and of health economics. Cardiovascular diseases (CVDs) are the most common disorders affecting most of the productive age group. Therefore, an attempt has been made to determine medication adherence using the Morisky medication adherence scale-4 (MMAS-4) and its determinants in women patients with CVD.

Material and Methods A Hospital based cross-sectional study was conducted at outpatient block of General Medicine and Cardiology departments which included 175 women patients who were already diagnosed with CVD (with at least 3 months of medication) and those who were willing to give informed consent for participation. Semi structured questionnaire was used which consisted of sociodemographic variables, MMAS-4 to measure the level of adherence and various determinants such as socioeconomic, patient related, condition related, therapy related, and health system related.

Results Prevalence of medication adherence among women of CVDs using MMAS-4 was high, medium, and low among 39,51 , and $10 \%$, respectively. Significant determinants found were socioeconomic, patient related, health system, and therapy related.

Conclusion Low-to-medium medication adherence was found to be high which needs to be addressed to reduce the morbidity and mortality associated with CVDs.

\section{Introduction}

Medication adherence is defined by the World Health Organization (WHO) as "the degree to which the person's behavior corresponds with the agreed recommendations from a health care provider. ${ }^{1}$ This is influenced by multiple factors which is clearly depicted in multidimensional adherence model by WHO with five dimensions including social and economic, patient, condition related, therapy related, and health care system factors. ${ }^{2}$ Effects of poor medication

published online

December 31, 2020
DOI https://doi.org/ 10.1055/s-0040-1708573. adherence results in disease progression with its complications such as myocardial infarction and stroke, increased out-of-pocket expenditure for emergency hospitalization, reduced quality of life, and increased mortality. ${ }^{3}$ Out of the 17 million premature deaths (under the age of 70 years) due to noncommunicable diseases, $82 \%$ occurred in low- and middle-income countries, of which $37 \%$ were contributed by cardiovascular diseases (CVDs). ${ }^{4}$ In addition, the average age of CVD patients in India is about a decade lower than in the Western world, with a greater proportion experiencing 


\section{Abstract Image}

\section{Medical adherence and its determinants among women of cardiovascular} disease of tertiary care Hospital, Hyderabad

\section{INTRODUCTION - CVDs}

Leading cause of global deaths

$\checkmark$ Pore adherence to long term therapies $\rightarrow$ Severely compromises the effectiveness of treatment

$\checkmark$ Medication adherence \& its determinants play an important role in assessing the mortality and morbidity caused by CVDs

Materials \& Methods
$>$ Study Type - Hospital based Cross section study
$>$ Place - OPD of General Medicine \& Cardiology
$>$ Population - 175 women patients Diagnosed with
CVD
$>$ Analysis - Using Morisky Scale (MMAS - 4)
$>$ Questionnaire - Determinants (Fig.)
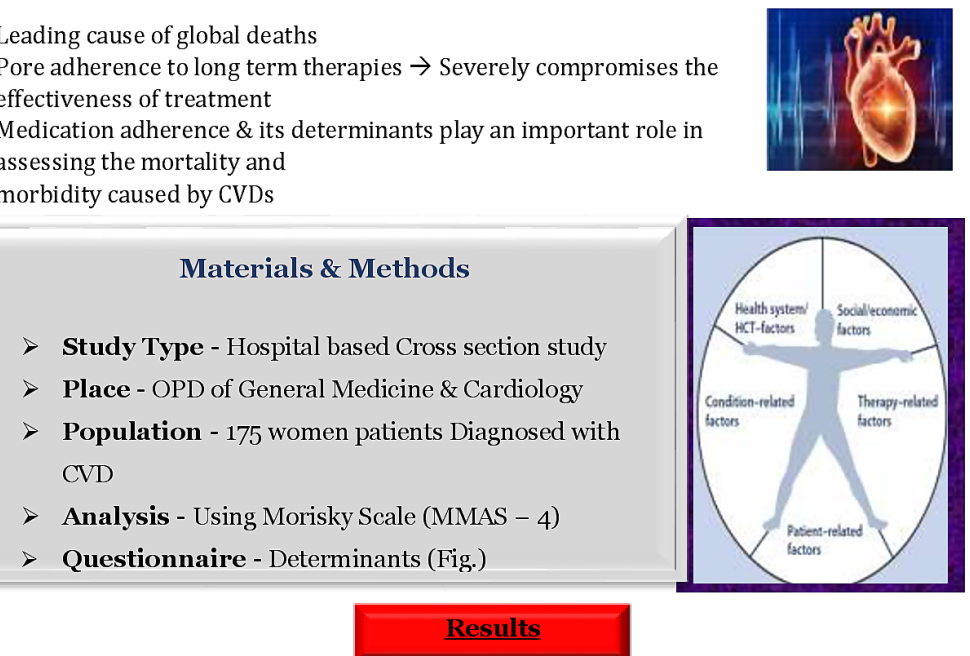

\section{Results}

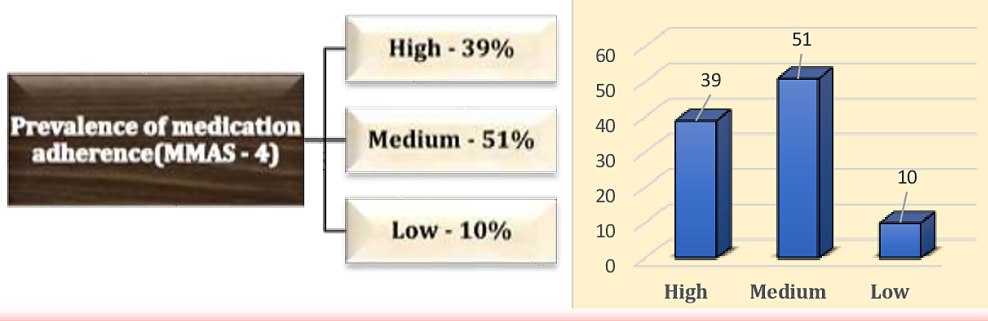

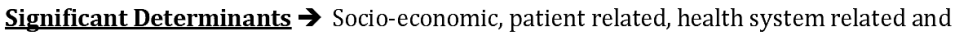
therapy related

\section{$\underline{\text { Conclusion }}$}

A low to medium medication adherence was found to be high which needs to be addressed to reduce the morbidity and mortality associated with CVDs

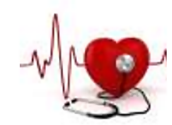

myocardial infarction and poor outcomes. ${ }^{5}$ Recommended guidelines to reduce the morbidity and mortality associated with CVDs were lifestyle modifications such as maintenance of body weight, increased physical activity, dietary modifications and cessation of smoking along with prescribed intake of antihypertensive, antiplatelets, and statins. Among these the most critical determinant is adherence to medication at the secondary level of prevention. ${ }^{6}$

Nonadherence to cardiovascular medications has become increasingly documented across patient populations and cardiovascular drug classes throughout the world. A metaanalysis of 20 studies involving 376,162 patients illustrates a 
nonadherence prevalence of $43 \%$ throughout multiple drug classes, as measured by pharmacy refill data. ${ }^{7}$ This is well supported by WHO, where medication adherence accounts to $50 \%$ in developed countries and the situation seems to be much worse in developing countries, like India, due to poor access to health care services, poor affordability, and poor knowledge of the disease outcome. ${ }^{8}$ One of the most common method used to measure this medication adherence was self-reported medication taking behavior using validated the Morisky medication adherence scale- 4 (MMAS-4). ${ }^{9}$

Indian studies have also found varied prevalence with low adherence among women..$^{10-12}$ Gender specific could be attributed more to patient-related determinants wherein the chances of forgetfulness to take medicine could be high due to her increased roles and responsibilities along with other factors. There is also paucity of studies, specifically among women in our set up to understand the interplay of various determinants influencing the medication adherence. This early identification of the determinants which are amenable for modification can be addressed in clinical practice to reduce the poorer outcome. Therefore, this study has been taken up especially among women with an objective to ascertain the magnitude of level of medication adherence and also assess various determinants influencing its level.

\section{Material and Methods}

A hospital-based cross-sectional study was conducted at a tertiary care hospital located at Hyderabad. The study includes women patients who were already diagnosed with CVD, such as coronary artery diseases, hypertension, and stroke (with at least 3 months of medication), above the age of 18 years, and those who gave written consent for participation and attending as outpatients (OPD). Those who were not willing to be a part of this study, below the age of 18 years, pregnant, and lactating women were excluded. The sample size was calculated using the formulae $4 P Q / L^{2}$ with prevalence assumed as $50 \%$ from previous studies conducted in India ${ }^{10}$ and was rounded to 175 . Simple random sampling method was adopted to select the women cases attending general medicine and cardiology OPD during 3 months of study period. Institutional ethical committee permission was obtained prior to the study. The Purpose of the study was explained and written informed consent was obtained from all the participants before administrating the questionnaire. Pilot study was conducted prior to the study and modifications were done in the questionnaire accordingly. Semistructured questionnaire was used consisting of sociodemographic variables, family history of hypertension/diabetes/coronary artery disease, reasons of good adherence and adherences, and personal habits. Clinical variables, such as type and duration of CVD, type and duration of medications, used by the patient were also detailed. The MMAS- 4 was used to estimate the level of medication adherence which consisted of four questions. For each question with answer as "Yes" was allotted one point and with answer "No" was allotted zero points. Based on the score from these four questions, they were classified as high adherence with score of 0 , medium adherence with score of 1 or 2 , and low adherence with score of 3 or 4 . Comprehensive WHO multidimensional adherence model classified the factors/determinants, such as socioeconomic determinants, which included poverty, unemployment, illiteracy, limited drug supply, and cost of medicines which were evaluated by probing questions like out of pocket expenditure. Patient-related determinants were assessed through their personal unwillingness, forgetfulness, taken alternative medicine, such as native medicine, ayurvedic, unani, or homeopathy medicines, and then felt better and stopped the medicines on their own. Health system-related determinants included irregular supply of drugs from the government, irregular follow-up and were not informed by the physician to continue as a life-long medication. Therapy-related determinants are long duration of treatment, too many drugs to be taken, fear of dependency on drugs, side-effects of drugs, unknown about the disease, and its complications. Condition-related determinants included usage of tobacco, alcohol, and other comorbidities associated obtained from previous reports and medications being taken were evaluated in the questionnaire.

\section{Statistical Analysis}

Data were entered in Microsoft excel 2018 and analyzed using Open Epi calculator to determine the association between various determinants and medication adherence using row by column table. Descriptive statistics are analyzed as frequency and mean \pm standard deviation. Chi-square test was used to predict the association between various determinants and medication adherence.

\section{Results}

Our study included 175 women above the age of 18 years diagnosed with any of the CVDs such as hypertension, coronary artery disease, and stroke. Women diagnosed with hypertension, coronary artery disease, and stroke were 145 (83\%), 18 (10\%), and 12 (7\%), respectively (-Fig. 1).

\section{Sociodemographic Characteristics}

Our study consisted Hindus 133 (76\%), Muslims 30 (17\%) and Christians 12 (7\%). As per their educational status, they were illiterates 110 (63\%), primary school attainment 30 (17\%), middle school $12(7 \%)$, high school $18(10.4 \%)$, graduate/ postgraduate $4(2 \%)$, and professional $1(0.6 \%)$.

Their occupational status is as follows: unemployed 136 (77\%), unskilled 23(14\%), semiskilled 8(5\%), skilled 7(3\%), and professional $1(1 \%)$. Among them, unmarried, married, and widow were 3(1.7\%), 136 (78\%), and 36 (20.3\%). Again, 138 (79\%) belonged to nuclear family, 30 (17\%) belonged to joint family, and 7(4\%) belonged to extended family. Mean age was found to be $54.13 \pm 10.53$ years. As per modified B.G. ${ }^{13}$ Prasad's classification, 34 (19\%) belonged to upper class, 67 (38\%) belonged to upper middle class, 51 (31\%) belonged to middle class, 23 (13\%) belonged to lower middle class, and $0(0 \%)$ belonged to lower class.

Family history of hypertension was found among 80 (44\%) and coronary artery disease among 7 (4\%) of our study participants. Alcohol usage was found among $9(5 \%)$ and tobacco usage 


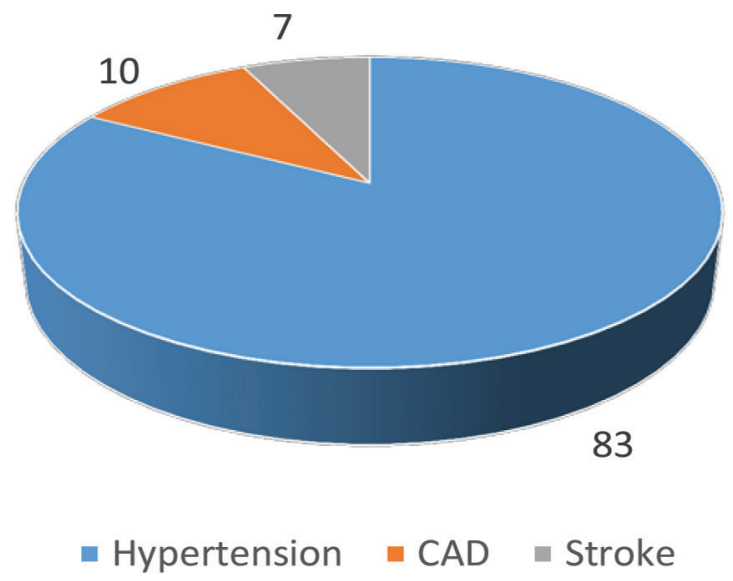

Fig. 1 Distribution of study subjects according to CVD. CVD, cardiovascular diseases.

was found among 14 (8\%) women. Mean duration of taking medication for any of the CVD was $5.33 \pm 4.85$ years.

Prevalence of Medication Adherence among Women of Cardiovascular Diseases Using the Morisky Scale

Prevalence of medication adherence among women of CVDs using MMAS-4 was found high, medium, and low among 68 (39\%), 89 (51\%), and 18 (10\%), respectively (-Fig. 2).

\section{Common Reasons for Good Adherence}

Most common reasons for good adherence to medicines were the multiple responses that included the good doctor-patient relationship 118 (68\%), followed by good knowledge of disease and complications 113 (65\%), and regular follow-up 114 (65\%).

\section{Common Reasons for Poor Adherence}

The Most common reasons for poor adherence to medicines were multiple responses and included forgetfulness 20 (11\%), poverty 16 (9.1\%), irregular supply of drugs 14 (8\%), and limited supply among 13 (7.4\%).

\section{Various Determinants of Medication Adherence among Study Patients}

Various determinants of medication adherence were classified into four main categories such as socioeconomic, patient related, health system related, and therapy related.

\section{Socioeconomic Determinants}

Poverty, unemployment, illiteracy, limited supply of drugs, and high cost of medicines were the major determinants and statistically significant with low-medication adherence in our study (-Table 1). Out-of-pocket expenditure was found to be Rupees. 415/- as a mean and maximum amount was up to Rupees. 4,000/- among our participants.

\section{Patient-Related Determinants}

Statistically significant association was found with unwillingness of patients to take medicines and forgetfulness of patients on analysis with low-medication adherence (-Table 2). Other reasons, such as symptoms subsided/felt better so stopped medicines and taking alternative medicine, were not associated with medication adherence.

\section{Health System-Related Determinants}

Irregular supply of drugs from government center, not informed by doctor about continuing medicines, and life-long and irregular follow-up were statistically significant with low-medication adherence in our study patients (- Table $\mathbf{3}$ ).

\section{Therapy-Related Determinants}

Long duration of treatment, adverse effects of drugs, and no knowledge of complications were statistically significant with low-medication adherence (-Table 4). Other factors, such as complex treatment regimen/multiple drugs, fear of dependence, and understanding/perceptions of disease as

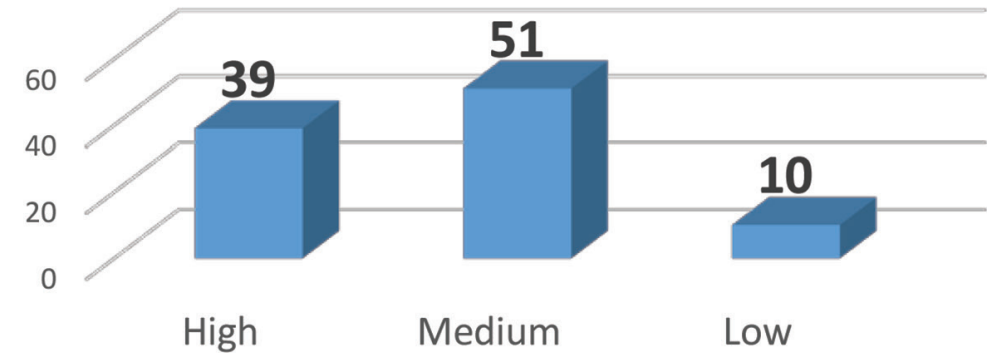

Fig. 2 Prevalence of medication adherence among study subjects using the Morisky medication adherence scale-4 (MMAS-4). 
Table 1 Association of socioeconomic determinants with medication adherence

\begin{tabular}{|c|c|c|c|c|c|}
\hline Socioeconomic determinants & & $\begin{array}{l}\text { Good } \\
\text { adherence } \\
n=68(\%)\end{array}$ & $\begin{array}{l}\text { Medium } \\
\text { adherence } \\
n=89(\%)\end{array}$ & $\begin{array}{l}\text { Low } \\
\text { adherence } \\
n=18(\%)\end{array}$ & $\begin{array}{l}\text { Total } \\
n=175(\%)\end{array}$ \\
\hline \multirow[t]{3}{*}{ Poverty } & Yes & $0(0)$ & $5(45)$ & $11(55)$ & $16(100)$ \\
\hline & No & $68(43)$ & $84(53)$ & $7(4 \%)$ & $159(100)$ \\
\hline & \multicolumn{5}{|c|}{$X^{2}=66.69 ; p=0.0001$} \\
\hline \multirow[t]{3}{*}{ Unemployment } & Yes & $0(0)$ & $0(0)$ & $4(100)$ & $4(100)$ \\
\hline & No & $68(40)$ & $89(52)$ & $14(8)$ & $171(100)$ \\
\hline & \multicolumn{5}{|c|}{$X^{2}=35.70 ; p=0.0004$} \\
\hline \multirow[t]{3}{*}{ Illiteracy } & Yes & $0(0)$ & $0(0)$ & $6(100)$ & $6(100)$ \\
\hline & No & $68(40)$ & $89(53)$ & $12(7)$ & $169(100)$ \\
\hline & \multicolumn{5}{|c|}{$X^{2}=54.19 ; p=0.0003$} \\
\hline \multirow[t]{3}{*}{ Limited drug supply } & Yes & \multicolumn{2}{|l|}{$\begin{array}{l}0(0) \\
3(23)\end{array}$} & $10(77)$ & $13(100)$ \\
\hline & No & $68(42)$ & $86(53)$ & $8(5)$ & $162(100)$ \\
\hline & \multicolumn{5}{|c|}{$X^{2}=68.27 ; p=0.0001$} \\
\hline \multirow[t]{3}{*}{ High cost } & Yes & $0(0)$ & $3(37)$ & $5(63)$ & $8(100)$ \\
\hline & No & $68(41)$ & $86(51)$ & $13(8)$ & $167(100)$ \\
\hline & \multicolumn{5}{|c|}{$X^{2}=25.72 ; p=0.001$} \\
\hline
\end{tabular}

Table 2 Association of patient-related determinants with medication adherence

\begin{tabular}{|c|c|c|c|c|c|}
\hline Patient-related determinants & & $\begin{array}{l}\text { Good } \\
\text { adherence } \\
n=68(\%)\end{array}$ & $\begin{array}{l}\text { Medium } \\
\text { adherence } \\
n=89(\%)\end{array}$ & $\begin{array}{l}\text { Low } \\
\text { adherence } \\
n=18(\%)\end{array}$ & $\begin{array}{l}\text { Total } \\
n=175(\%)\end{array}$ \\
\hline \multirow[t]{3}{*}{ Unwillingness } & Yes & $0(0)$ & $0(0)$ & $5(100)$ & $5(100)$ \\
\hline & No & $68(40)$ & $86(52)$ & $13(8)$ & $170(100)$ \\
\hline & \multicolumn{5}{|c|}{$X^{2}=44.89 ; p=0.0001$} \\
\hline \multirow[t]{3}{*}{ Forgetfulness } & Yes & $0(0)$ & $6(30)$ & $14(70)$ & $20(100)$ \\
\hline & No & $68(44)$ & $83(53)$ & $4(3)$ & $155(100)$ \\
\hline & \multicolumn{5}{|c|}{$X^{2}=88.25 ; p=0.000001$} \\
\hline
\end{tabular}

Table 3 Association of health system-related determinants with medication adherence

\begin{tabular}{|c|c|c|c|c|c|}
\hline Health system determinants & & $\begin{array}{l}\text { Good } \\
\text { adherence } \\
n=68(\%)\end{array}$ & $\begin{array}{l}\text { Medium } \\
\text { adherence } \\
n=89(\%)\end{array}$ & $\begin{array}{l}\text { Low } \\
\text { adherence } \\
n=18(\%)\end{array}$ & $\begin{array}{l}\text { Total } \\
n=175(\%)\end{array}$ \\
\hline \multirow{3}{*}{$\begin{array}{l}\text { Irregular supply of drugs from } \\
\text { government center }\end{array}$} & Yes & $0(0)$ & $2(14)$ & $12(86)$ & $14(100)$ \\
\hline & No & $68(42)$ & $87(54)$ & $6(4)$ & $161(100)$ \\
\hline & \multicolumn{5}{|c|}{$X^{2}=94.08 ; p=0.0001$} \\
\hline \multirow{3}{*}{$\begin{array}{l}\text { Not informed by doctor about } \\
\text { continuing medicines life-long }\end{array}$} & Yes & $0(0)$ & $1(33)$ & $2(67)$ & $3(100)$ \\
\hline & No & $68(41)$ & $88(51)$ & $16(10)$ & $172(100)$ \\
\hline & \multicolumn{5}{|c|}{$X^{2}=10.81 ; p=0.005$} \\
\hline \multirow[t]{3}{*}{ Irregular follow-up } & Yes & $0(0)$ & $2(20)$ & $8(80)$ & $10(100)$ \\
\hline & No & $68(41)$ & $87(52)$ & $10(7)$ & $165(100)$ \\
\hline & \multicolumn{5}{|c|}{$X^{2}=55.86 ; p=0.0001$} \\
\hline
\end{tabular}


Table 4 Association of therapy-related determinants with medication adherence

\begin{tabular}{|c|c|c|c|c|c|}
\hline Therapy-related determinants & & $\begin{array}{l}\text { Good } \\
\text { adherence } \\
n=68(\%)\end{array}$ & $\begin{array}{l}\text { Medium } \\
\text { adherence } \\
n=89(\%)\end{array}$ & $\begin{array}{l}\text { Low } \\
\text { adherence } \\
n=18(\%)\end{array}$ & $\begin{array}{l}\text { Total } \\
n=175(\%)\end{array}$ \\
\hline \multirow[t]{3}{*}{ Long duration of treatment } & Yes & $0(0)$ & $2(22)$ & $7(78)$ & $9(100)$ \\
\hline & No & $68(41)$ & $87(52)$ & $11(7)$ & $166(100)$ \\
\hline & \multicolumn{5}{|c|}{$X^{2}=47.23 ; p=0.0001$} \\
\hline \multirow[t]{3}{*}{ Adverse effects of drugs } & Yes & $0(0)$ & $0(0)$ & $3(100)$ & $3(100)$ \\
\hline & No & $68(39)$ & $89(52)$ & $15(9)$ & $172(100)$ \\
\hline & \multicolumn{5}{|c|}{$X^{2}=26.62 ; p=0.001$} \\
\hline \multirow[t]{3}{*}{ No knowledge of complications } & Yes & $0(0)$ & $2(33)$ & $4(67)$ & $6(100)$ \\
\hline & No & $68(40)$ & $87(52)$ & $14(8)$ & $169(100)$ \\
\hline & \multicolumn{5}{|c|}{$X^{2}=21.99 ; p=0.0001$} \\
\hline
\end{tabular}

life-threatening or dangerous, were neither significant nor associated in our study.

\section{Condition-Related Determinants}

Comorbidities associated were diabetes mellitus among $41(23 \%)$ and thyroid disorders among 15 (8\%), but statistical significance cannot be established with these condition-related factors along with their personal habits, such as alcohol and tobacco usage, due to their small number.

\section{Discussion}

In our study, approximately $61 \%$ of the women were found to have low-to-medium level of medication adherence for the secondary prevention of CVDs at a tertiary care hospital of Hyderabad. Thakur et al, in their study at Chandigarh, found $48.1 \%$ of women to have low-to-medium level of medication adherence for the coronary artery disease using MMAS- $8 .{ }^{10}$ In their study at Puducherry, to assess adherence among hypertensive patients, Shankar et al also found $63 \%$ of women to have reported nonadherence using MMAS having seven question with four-point response format. ${ }^{4}$ Ramli et al, in their study at primary health care clinics of Malaysia, found $43.7 \%$ of women with hypertension to have nonadherence to medication using MMAS-8. ${ }^{5}$ All these variations can be explained by varying sociodemographic regions and also varying scales used to measure the adherence.

Complex interplay of multiple factors determine adherence to medication. In the present study, it was found that the most common reasons for poor adherence were forgetfulness (11\%), poverty (9.1\%), irregular supply (8\%), and limited supply of drugs (7.4\%). Statistically significant association was found with socioeconomic determinants which included poverty, unemployment, illiteracy, limited drugs supply, and high cost of medicines. Among the patient-related determinants, unwillingness and forgetfulness were found to be statistically significant. Health system-related determinants were irregular supply, not informed by the physician about life-long intake of medicines, and irregular follow-up. Therapy-related determinants, such as long duration, adverse events of drugs, and no knowledge about complications, were also found statistically significant among our study patients.

Similar findings were found by Awad et al, in their study among Sudanese, where the most common reasons for low-medication adherence were high cost of drugs, polypharmacy, lack of pharmacist's communication with them regarding the instructions and importance of taking the drug, lack of physician's communication with them regarding their illness, side-effects associated with their medications, and irregular availability of the drugs in their areas. ${ }^{6}$ Van der Laan et al among the Netherland patients also found older age, forgetfulness, too many pills, and insufficient knowledge on what to do when a dose is forgotten as the common factors for determining medication adherence. ${ }^{7}$ In Indian set up, similar findings were also reported by Gupta et al with main factors as forgetfulness and absence of symptoms of hypertension, drugs side-effects, and out of supply as reasons for their medication adherence which accounted to $20 \%$ medium and 21.4\% low-medication adherence. ${ }^{8}$ Socioeconomic determinants seem to play a predominant role in low- and middle-income countries compared with high-income countries. And also the self-reported behavior of noncompliance needs to be changed to reduce the morbidity and mortality.

\section{Conclusion}

Low-to-medium medication adherence was found to be more than half among our study patients which needs to be addressed by targeting the common determinants, such as forgetfulness, which can be reduced by putting reminders in mobile phones, creating health education about the importance of intake of drugs, and thereby preventing complications with regular follow-up, and also the physician's utmost need to explain the life-long intake along with lifestyle modifications. 


\section{Audio}

Audio file for this article is available at https://doi.org/ $10.1055 / \mathrm{s}-0040-1708573$.

\section{Conflicts of Interest}

None declared.

\section{Funding}

Students Prajwalika Scholarship program 2019, Women in Cardiology and related sciences association.

\section{Acknowledgments}

We would extend our sincere thanks to Dr. Rajiv, Assistant Professor, Department of General Medicine and Dr. Sai, Cardiologist for their kind cooperation in the outpatient block. Also to all our study patients who gave their consent to participate in our project.

We thank our guide Dr. Sudha Bala and all the participants for extending the support.

\section{References}

1 Rand CS. Measuring adherence with therapy for chronic diseases: implications for the treatment of heterozygous familial hypercholesterolemia. Am J Cardiol 1993;72(10):68D-74D

2 Sabate E; World Health Organization, Adherence to Long Term Therapies. Evidence for Action. Geneva, Switzerland: World Health Organization; 2003

3 Osterberg L, Blaschke T. Adherence to medication. N Engl J Med 2005;353(5):487-497

4 World Health Organization. Cardiovascular diseases (CVDs). Fact sheets. Available at: https://www.who.int/news-room/factsheets/detail/cardiovascular-diseases-(cvds) Accessed October 4, 2019

5 Xavier D, Pais P, Devereaux PJ, et al; CREATE registry investigators. Treatment and outcomes of acute coronary syndromes in India (CREATE): a prospective analysis of registry data. Lancet 2008;371(9622):1435-1442

6 Smith SC Jr, Allen J, Blair SN, et al; AHA; ACC; National Heart, Lung, and Blood Institute. AHA/ACC guidelines for secondary prevention for patients with coronary and other atherosclerotic vascular disease: 2006 update endorsed by the National Heart, Lung, and Blood Institute. J Am Coll Cardiol 2006;47(10):2130-2139
7 Naderi SH, Bestwick JP, Wald DS. Adherence to drugs that prevent cardiovascular disease: meta-analysis on 376,162 patients. Am J Med 2012;125(9):882-7.e1

8 Akeroyd JM, Chan WJ, Kamal AK, Palaniappan L, Virani SS. Adherence to cardiovascular medications in the South Asian population: a systematic review of current evidence and future directions. World J Cardiol 2015;7(12):938-947

9 Morisky DE, Green LW, Levine DM. Concurrent and predictive validity of a self-reported measure of medication adherence. Med Care 1986;24(1):67-74

10 Thakur JS, Vijayvergiya R, Jaswal N, Ginsburg A. Assessment and barriers to medication adherence for secondary prevention of cardiovascular disease among patients with coronary artery disease in Chandigarh, India. Int J Non-Commun Dis 2016;1:37-41

11 Rao CR, Kamath VG, Shetty A, Kamath A. Treatment compliance among patients with hypertension and type 2 diabetes mellitus in a coastal population of southern India. Int J Prev Med 2014;5(8):992-998

12 Venkatachalam J, Abrahm SB, Singh Z, Stalin P, Sathya GR. Determinants of patient's adherence to hypertension medications in a rural population of Kancheepuram district in Tamil Nadu, South India. Indian J Community Med 2015;40(1):33-37

13 Pandey VK, Aggarwal P, Kakkar R. Modified BG Prasad's Socio-economic Classification-2018: The need of an update in the present scenario. Indian J Comm Health 2018;30(1):82-84

14 Shankar SP, Suman Babu ISS, Ramya N. A study on usefulness of modified medication adherence scale in assessing adherence among hypertensive patients. Perspect Clin Res 2019;10(4):163-167

15 Ramli A, Ahmad NS, Paraidathathu T. Medication adherence among hypertensive patients of primary health clinics in Malaysia. Patient Prefer Adherence 2012;6:613-622

16 Awad A, Osman N, Altayib S. Medication adherence among cardiac patients in Khartoum State, Sudan: a cross-sectional study. Cardiovasc J Afr 2017;28(6):350-355

17 van der Laan DM, Elders PJM, Boons CCLM, Nijpels G, Hugtenburg JG. Factors associated with nonadherence to cardiovascular medications: a cross-sectional study. J Cardiovasc Nurs 2019;34(4):344-352

18 Gupta MC, Bhattacharjee A, Singh H. Evaluation of medication adherence patterns amongst anti-hypertensive drug users in a tertiary care hospital in north India. Int J Basic Clin Pharmacol 2016;5:2261-2266 